\title{
Infertilidad tubárica e infección genital por Chlamydia trachomatis-Ureaplasma urealyticum
}

\author{
José Urdaneta M., $P h D^{1}$, Eliana Cantillo $H^{2,3}$, Andrés Alarcón S., $M S^{2}{ }^{2}$, Abdala Kara- \\ me H., MSc ${ }^{2}$, Jenny Salazar de Acosta, $P h D^{1}$, Zoila Romero A., $P h D^{1}$, Nasser Baabel \\ Romero ${ }^{a}$, Endrina Mujica $A^{a}$
}

${ }^{1}$ Universidad del Zulia, Facultad de Medicina, Escuela de Bioanálisis, Departamento de Morfofisiopatología; ${ }^{2}$ Instituto Venezolano de Fertilidad; 3Programa de Posgrado de Obstetricia y Ginecología, Facultad de Medicina, Universidad del Zulia. Maracaibo, Venezuela.

${ }^{a}$ Alumno, Escuela de Medicina, Universidad del Zulia, Venezuela.

\section{RESUMEN}

Objetivo: Establecer la asociación entre la infertilidad tubárica y la infección cervical por Chlamydia trachomatis (CT) o Ureaplasma urealiticum (UU), en mujeres infértiles. Métodos: Investigación comparativa y aplicada, con diseño de tipo no experimental, de casos y controles, contemporáneo transeccional y de campo, que incluyó 60 mujeres, separadas en dos grupos pareados de acuerdo si eran infértiles (casos) o fértiles (controles), a las cuales se les tomó una muestra de hisopado endocervical para el diagnóstico molecular de CT o UU y se les realizó una histerosalpingografía para evaluar la permeabilidad de las trompas uterinas. Resultados: Se detectó una prevalencia en mujeres infértiles y fértiles de infección por CT o UU del $18 \%$ y $35 \%$, respectivamente; siendo mayor entre las mujeres infértiles, diferencia significativa solo para UU $(p<0,05)$. Se detectó una mayor permeabilidad tubárica en las pacientes fértiles que en las infértiles ( $80 \%$ vs. $40 \%)$, siendo el compromiso tubárico mayor en las pacientes infértiles $(p<0,05)$. Al asociar el diagnóstico de CT o UU con los resultados de la histerosalpingografía se constató que la detección de uno de estos microorganismos aumentaba casi 3 o 5 veces más la probabilidad de presentar obstrucción tubárica, respectivamente, diferencias no significativas $(p>0,05)$. Conclusión: Una gran parte de las mujeres infértiles presentan infección por CT o UU, patógenos de transmisión sexual que pudiesen tener responsabilidad en el daño tubárico.

\section{PALABRAS CLAVE: Chlamydia trachomatis, infección genital, infertilidad tubárica, Ureaplasma urealyticum}

\section{SUMMARY}

Objective: To establish the association between tubal infertility and cervical infection by Chlamydia trachomatis (CT) or Ureaplasma urealyticum (UU) in infertile women. Methods: A comparative, and applied research with a non-experimental, case-control, contemporary-transactional and field design, including 60 women, separated into two groups matched according whether they were infertile (cases) or fertile (controls), in which was took a sample of endocervical swabs for molecular diagnosis of CT or UU and underwent hysterosalpingography to assess the permeability of the fallopian tubes. Results: It was detected in infertile and fertile women a prevalence of CT or UU infection of $18 \%$ and $35 \%$, respectively; being higher detection among infertile women, although this difference was significant only for $U U(p<0.05)$. Also detected more tubal permeability in fertile patients that in infertile ( $80 \%$ vs. $40 \%)$, being higher in engagement tubal in infertility patients $(p<0.05)$. By associating the diagnosis of both CT and UU with hysterosalpingography's 
results found that the diagnosis of one of these microorganisms increased almost 3 to 5 times more likely to have obstruction of the fallopian tubes, respectively; although this higher risk doesn't showed significance ( $p>0.05)$. Conclusion: A large proportion of infertile women have CT or UU infection, sexually transmitted pathogens that might have tubal damage liability.

\section{KEY WORDS: Chlamydia trachomatis, genital infection, tubal infertility, Ureaplasma urealyticum}

\section{INTRODUCCIÓN}

La especie humana es una de las que presenta mayores dificultades para la procreación, una mujer sin problemas de fertilidad pierde dos de cada tres embriones fecundados (1); comparado con otras especies, el ser humano es altamente ineficiente en términos de reproducción, la tasa de fertilidad por ciclo es de alrededor de $20 \%$ y la de embarazos acumulados en las parejas con fertilidad probada es aproximadamente del $90 \%$ después de doce meses y del $94 \%$ luego de dos años (2).

Alrededor del mundo existen aproximadamente ochenta millones de parejas con problemas de fertilidad o de concepción, es decir, unas quince de cada cien parejas no logran la fertilidad o no pueden llevar a cabo un embarazo a término; se calcula que de éstas hasta un $8 \%$ de los casos son debidos a infertilidad primaria, mientras que la infertilidad secundaria tiene un porcentaje del $35 \%$, significativamente mayor (3). Las tasas de infertilidad varían dramáticamente desde $5 \%$ en países desarrollados hasta más del $30 \%$ en el África Subsahariana (3); en tanto que en el Reino Unido se calcula que una de cada 7 parejas son infértiles (4) y en los Estados Unidos de Norteamérica se estima que afecta a 10$15 \%$ de las parejas (5), en la actualidad más del $1 \%$ de todos los nacimientos en ese país son por medio de tecnologías de reproducción asistida (6).

No hay evidencias provenientes de estudios poblacionales que sugieran una mayor incidencia de parejas infértiles, sin embargo, aparentemente en los últimos años sí ha habido un aumento del número de consultas a las clínicas de infertilidad, el cual podría ser debido a cuatro factores: el aumento en la edad promedio a la cual la mujer desea quedar embarazada, las alteraciones en la calidad del semen, cambios en la conducta sexual y la eliminación tanto de la mayoría de los tabúes sobre la fertilidad como la mayor difusión de los tratamientos disponibles (2).

De acuerdo con Ortiz y cols (7), la infección genital es la causa más importante de infertilidad a nivel mundial; siendo reconocida la infección por bacterias de transmisión sexual como un factor predisponente para el desarrollo de infertilidad tubárica, sobre todo la colonización por Chlamydia trachomatis (CT) o Neisseria gonorrhoeae (NG) (8); aunque también se ha involucrado bacterias de la clase Mollicutes como Ureaplasma ureolyticum
(UU) o Mycoplasma hominis (MH).

Son numerosas las evidencias que demuestran que las infecciones genitales en la mujer conducen a enfermedad inflamatoria pélvica, la cual genera daños permanentes a las trompas de Falopio, al útero y a los tejidos circundantes, estos daños tienen consecuencias a mediano y corto plazo que provocan infertilidad (7). Al respecto, se ha vinculación la infertilidad, sobre todo aquella por factor tubárico, a la infección por CT, patógeno que ha podido aislarse en la mayoría de las mujeres con infertilidad tubárica; detectándose títulos elevados de anticuerpos anti-C trachomatis en más del $70 \%$ de las mujeres con oclusión de las trompas uterina (9).

Dada una prevalencia en todo el mundo de 50 millones de nuevas infecciones por CT en mujeres cada año, un grupo clínicamente significativo de las mujeres infectadas por este patógeno podrían encontrarse en riesgo de secuelas como la infertilidad (10). Tan sólo en los Estados Unidos de América ocurren cerca de 3 millones de casos anuales de infecciones por CT (11), constituyendo un importantes problema de salud pública, sobre todo en mujeres jóvenes sexualmente activas, a las cuales se les ha recomendado la realización anual de pruebas de pesquisa $(12,13)$.

Aunque las infecciones del tracto urogenital por CT es común y ha sido reconocida como una causa importante de infertilidad tubárica, los mecanismos patogénicos del daño en las trompas inducido por CT siguen siendo desconocidos, y no existen para la fecha vacunas eficaces disponibles (9), además que un $75 \%$ de las mujeres y un $50 \%$ de los hombres infectados son asintomáticos, lo cual puede retrasar el diagnóstico y aumentar el riesgo de secuelas a largo plazo (14). Esta circunstancia se agrava puesto de que a pesar de los avances en las técnicas disponible para el diagnóstico de éste patógeno, en la mayoría de los países, incluyendo a Venezuela, se presenta la misma problemática relacionada con la inexistencia de métodos de diagnóstico sensibles, rápidos y viables, lo que se traduce en un problema mayor, debido a que las mujeres infectadas, al no ser diagnosticadas, no reciben tratamiento oportuno, desarrollando complicaciones como abortos e infertilidad (15).

Asimismo, en los países en desarrollo, los datos sobre la prevalencia de infecciones genitales por CT y sus secuelas, en especial la infertilidad tubárica, es escasa (16), encontrándose en la región de 
América Latina y el Caribe limitada a un pequeño número de estudios y a datos oficiales incompletos de los países de la región (17). En Venezuela y particularmente en el Estado Zulia, el diagnóstico de infecciones por CT o UU se basa fundamentalmente en la presencia o ausencia de manifestaciones clínicas, estrategia que presenta muchas limitaciones dado la alta tasa de infecciones asintomáticas por éste patógeno, motivo por el cual se desconoce la verdadera prevalencia de esta infección (18). Específicamente en la ciudad de Maracaibo se han reportado datos de prevalencia de infección por CT, detectada por diagnóstico molecular en mujeres con o sin sintomatología de infección genital, del $9,26 \%$ en instituciones del sector público (19) y del 7,7\% en clínicas privadas (20); mientras que en poblaciones rurales del Estado Zulia la prevalencia reportada ha sido del 10,14\% (18).

Por otra parte, a pesar de que UU ha sido descrito como productor de infecciones de transmisión sexual, su rol patógeno es todavía motivo de controversia debido a que pueden formar parte de la flora normal del tracto genital de mujeres sexualmente activas (21). Si bien el papel exacto de UU en pacientes con problemas de infertilidad no se ha dilucidado completamente y no está claro si contribuye significativamente a la infertilidad (22). Teniendo en cuenta que la infección por UU a menudo es concomitante con CT, la cual está relacionada con infertilidad debido a factor tubárico, la presencia de UU pudiese tener un papel en la infección subclínica y tuboperitoneal, por lo que se debe ofrecer el cribado de estas infecciones en las pacientes sometidas a una evaluación de infertilidad (23).

Como puede inferirse de lo anterior, no se conoce la verdadera prevalencia de estas infecciones entre las mujeres en edad fértil, que tendría una mayor relevancia entre las pacientes por problemas de fertilidad, surgiendo la necesidad de establecer la asociación entre la infertilidad tubárica diagnosticada mediante histerosalpingografía y la presencia de infección genital por CT o UU diagnosticada mediante técnicas moleculares de amplificación de ADN en mujeres que consultan por infertilidad en el Instituto Venezolano de Fertilidad, Maracaibo, Venezuela; durante el periodo comprendido entre octubre del 2011 a septiembre de 2012.

\section{METODOS}

Investigación de tipo comparativa y aplicada, adecuada a los propósitos del diseño no experimental, de casos y controles, contemporáneo transeccional y de campo, en la cual se tomó una muestra no probabilística e intencionada de 60 mujeres, divididas en dos grupos pareados de 30 sujetos cada uno, denominados: Grupo A (Casos) y Grupo B (Controles), conformados por mujeres infértiles y fértiles, respectivamente. Los casos se definieron como aquellas mujeres que presenten una inhabilidad para procrear después de un año de vida sexual activa no protegida por algún tipo de contracepción; mientras que los controles fueron toda aquella mujer que no cumplan con los criterios de la definición de caso y que haya culminado un embarazo con un producto viable, que hubiese asistido a la institución para solicitar algún método contraceptivo o la esterilización quirúrgica. Se tomó un control del respectivo centro asistencial por cada caso de infertilidad detectado.

De igual manera fueron fijados algunos criterios para la inclusión de las pacientes dentro del protocolo de estudio; estos criterios son:

1. Ausencia de embarazos después de un año de actividad sexual con pareja única y sin haber utilizado algún método contraceptivo para los casos.

2. Ausencia de enfermedades crónicas concomitantes.

3. Deseo voluntario de participar en la investigación y consentimiento informado del mismo.

Asimismo, fueron considerados los siguientes criterios de exclusión:

1. No cumplir con los criterios diagnósticos de infertilidad para los casos.

2. Uso de terapia antimicrobiana oral y/o vaginal en el último mes.

3. Aplicación de duchas vaginales o coito 48 horas previas a la obtención de la muestra cervical.

4. Negativa a participar en el estudio.

Para la ejecución de esta investigación, se seleccionaron en el Instituto Venezolano de Fertilidad de acuerdo con los criterios previamente establecidos, a las pacientes infértiles en la consulta de Infertilidad y en la consulta de planificación familiar a los controles fértiles. A cada paciente, se le informó sobre los propósitos de la investigación y previa firma del consentimiento informado fueron finalmente incluidas en la misma; esto con el propósito de dar cumplimiento con los lineamientos de la Declaración de Helsinki.

Seguidamente, las pacientes seleccionadas se citaron para efectuarles una evaluación ginecológica integral, donde por medio del interrogatorio médico se indagó acerca de datos socioeconómicos (edad, estado civil, procedencia, nivel socioeconómico), antecedentes clínicos y hábitos (co-morbilidades, consumos de drogas licitas e ilícitas), historia gineco-obstétrica (métodos de regulación de la fertilidad, sexarquía, número de parejas, antecedentes de abortos y gestaciones previas, infecciones de transmisión sexual previas); asentándose los datos pertinentes en el instrumento diseñado para tal fin. 
Asimismo, se realizó un examen clínico ginecológico en el cual fue evaluada la apariencia del cérvix (normal, ectropión, friabilidad, eritema), presencia de dolor a la movilización del cuello uterino, presencia de descarga vaginal con o sin dolor, tamaño y consistencia del útero, presencia de masas o dolor a la palpación de anexos y evaluación de las glándulas mamarias para descartar presencia de galactorrea. De igual manera, se tomaron muestras cervicovaginales para la realización del Papanicolau y para el diagnóstico molecular de CT y UU de acuerdo con el siguiente procedimiento:

(a) Toma de la muestra: Consistió en un hisopado del canal endocervical y se realizó empleando dos hisopos de algodón estériles. Para ello, las mujeres debían estar en periodo no menstrual y con abstinencia sexual previa de 48 horas. En posición ginecológica, se introdujo un especulo vaginal desechable de plástico sin lubricación, con fuente de luz externa se localizó el orificio cervical externo para proceder a la toma de un hisopado endocervical obtenido mediante rotación en la zona de transición escamo-columnar de la región endocervical. Este hisopo fue introducido en un tubo que contenía 1 $\mathrm{ml}$ de tampón fosfato salino utilizado como medio de transporte; estas muestras fueron trasladadas al laboratorio, mantenidas a $4^{\circ} \mathrm{C}$ y procesadas dentro de los primeros 3 días de su recepción, para evitar la contaminación de la muestra.

(b) Extracción del ADN: Para el procesamiento de las muestras y la extracción del ADN de CT y UU, se utilizó el procedimiento descrito previamente por Goessen y modificado por Arráiz y cols (19), el cual se describe a continuación: Se transfiere un volumen de $500 \mu \mathrm{L}$ de muestra a un tubo de $1,5 \mathrm{ml}$, se centrifuga a $14.000 \times \mathrm{g}$ y el sedimento se resuspende en $200 \mu \mathrm{L}$ de tampón de lisis $(50 \mathrm{mM}$ Tris$\mathrm{Hcl} \mathrm{pH} \mathrm{7,5,1 \%} \mathrm{Triton} \mathrm{X-100,} 1$ mM EDTA, $250 \mu \mathrm{g} /$ $\mathrm{ml}$ de proteinasa $\mathrm{K}$ ). Luego, se incuba la muestra a $56^{\circ} \mathrm{C}$ durante 2 horas y los lisados se extraen con fenol-cloroformo y se precipitan con etanol. EI ADN obtenido se resuspende en $30 \mu \mathrm{L}$ de tampón TE (10 $\mathrm{mM}$ Tris- $\mathrm{HCl}, \mathrm{pH} 8,1 \mathrm{mM}$ EDTA, $\mathrm{pH}$ 8).

(c) Amplificación por reacción en cadena de la polimerasa $(P C R)$ : Se utilizan $5 \mathrm{ml}$ de la muestra para ensayos de amplificación y detección de CT Y UU por reacción en cadena de la polimerasa (PCR), utilizando primers o iniciadores específicos para cada microorganismo. La amplificación se realizó mediante PCR múltiplex, utilizando el kit MPCR detection kit, CTR/UU/NG from Maxim Biotech, Inc., USA; el cual incluye cebadores dirigidos a secuencias específicas de ADN a partir de cada organismo, generando productos de amplificación de 364 pb para CTy 218 pb para UU. La mezcla de reacción y condiciones de amplificación de PCR fueron extraídos de las instrucciones dadas por el fabricante.

Para la detección de CT se llevaron a cabo reacciones de amplificación utilizando los oligonucleótidos CTP1 y CTP2 dirigidos a secuencias de un plásmido endógeno de CT, designado en este estudio RPCCTP; se utiliza una segunda reacción de PCR como ensayo confirmatorio utilizando oligonucleotidos SERO 1A y SERO 2A, específicos de secuencias del gen que codifica la proteína de membrana externa PME1 (RPCPME1). Con los fragmentos esperados para los ensayos RPCCTP y RPC PME1, respectivamente, se prepara una mezcla de reacción de un volumen final de $50 f \hat{E} L$ que consiste en $5 f \hat{E} L$ de tampon taq ADN polimerasa 10X (Promega), 1,5 mM MgCl2, $200 f$ ÊM de cada desoxirribonucleotido (dATP, dCTP, dGTP y dTTP), 20 pmoles de cada oligonucleótido. Se utiliza 0,25 $\mu \mathrm{L}$ de Taq ADN polimerasa 1U/I (PROMEGA) y $5 \mu \mathrm{L}$ de muestra de ADN para cada reacción.

Para la detección del genoma de UU, se utilizaron los cebadores U4 y U5, dirigidos al gen ureasa específica que genera un producto de $429 \mathrm{pb}$. La secuencia de los iniciadores son: U5 (5' CAA TCT GCT CGT GAA GTA TTA C 3') y U4 (5'ACG ACG TCC ATA AGC AAC T 3').

El programa de amplificación consistió en $4 \mathrm{mi}$ nutos a $94^{\circ} \mathrm{C}$ y 35 ciclos de amplificación de $1 \mathrm{mi}-$ nuto a $95^{\circ} \mathrm{C}, 1$ minuto a $54^{\circ} \mathrm{C}, 1,5$ minutos a $72^{\circ} \mathrm{C}$ y un paso final de amplificación a $72^{\circ} \mathrm{C}$ por $10 \mathrm{mi}-$ nutos; las muestras positivas se confirmaron por un segundo ensayo; donde se desnaturaliza a $95^{\circ} \mathrm{C}$ por 5 min y se programan 49 ciclos de amplificación de $1 \mathrm{~min}$ a $95^{\circ} \mathrm{C}, 1,5 \mathrm{~min}$ a $45^{\circ} \mathrm{C}, 1,5 \mathrm{~min}$ a $72^{\circ} \mathrm{C}$ y un paso final de extensión de 5 minutos a $72^{\circ} \mathrm{C}$. Las reacciones se realizaron en un termociclador MJ Research PTC- 100; una muestra se consideradó positiva cuando se obtenga amplificación de los fragmentos correspondientes para los dos ensayos. Se incluyó como control positivo para CT ADN aislado de muestras positivas tanto por inmunofluorescencia como por PCR y un cultivo control de UU; mientras que como control negativo se utilizó agua destilada.

(d) Electroforesis en gel de agarosa: Los productos de PCR se analizan en geles de agarosa al $2 \%$ (20 $\mu \mathrm{L}$ de cada muestra amplificada) y estos geles son teñidos con bromuro de etidio. El corrimiento electroforético se realizó a 70 volts durante 1,5 horas, para luego visualizarlos en un transiluminador ultravioleta a fin de revelar la presencia de ADN y finalmente fotografiarlos con una cámara digital Olympus C-4000 (photodocumentation system Digi Doc, UVP).

Las muestras de cada una de las participantes del estudio pruebas fueron procesadas en dos centros diferentes para tabular como resultados positivos, a las pacientes con identificación del genoma bacteriano en los dos ensayos practicados. Estos centros fueron el laboratorio de la Sección de Biología molecular del Centro de Investigaciones Endocrino-metabólicas "Dr. Félix Gómez" de la Facultad 
de Medicina de la Universidad del Zulia y el Centro Latinoamericano de Diagnóstico Genético Molecular (CELAGEM), ubicado en Bogotá, Colombia.

Los datos obtenidos se organizaron y recopilaron en una base de datos y se procedió a emplear el Paquete Estadístico para Ciencias Sociales (SPSS), versión 17; procediéndose a expresarlos mediante medidas de tendencia central: frecuencias absolutas, porcentajes, medias y desviación estándar (DE). Asimismo para la comparación entre los grupos se utilizó la prueba t de Student para los datos cuantitativos y la prueba del Chi cuadrado para los datos cualitativos; con una significación estadística de $p<0,05$. Por su parte, la estimación del riesgo de obstrucción tubárica ante la presencia de cada uno de los microorganismos estudiados, se realizó un análisis de regresión logística, expresando el riesgo en términos de Odds ratios (OR) con sus respectivos intervalos de confianza con un nivel de confianza del 95\% (IC95\%); la significancia estadística de estas razones fue determinada mediante la prueba del Chi cuadrado. Los resultados obtenidos de estos análisis se expresaron mediante el uso de tablas de distribución de frecuencias.

\section{RESULTADOS}

En primer lugar se presenta el análisis descriptivo cuantitativo de algunas de las principales características presentes en las pacientes incluidas en esta investigación. Como se observa en la Tabla I, la edad promedio de las pacientes infértiles fue de 37 años, mientras que en las fértiles la edad promedio fue de 36 años; por su parte, la edad de la menarquía y de la sexarquía estuvo en ambos grupos alrededor de los 12 y 19 años, respectivamente. Al indagar acerca del número de parejas o compañeros estables a lo largo de toda la vida, las pacientes de ambos grupos manifestaron entre 2 y 3 parejas; siendo el tiempo de convivencia con su pareja actual de aproximadamente de 5 años. El análisis cuantitativo pareado entre estas características de ambos grupos no evidenció diferencias estadísticas significativas entre las mujeres fértiles y las infértiles; lo cual, manifiesta la homogeneidad de la muestra.

En la Tabla II se presenta el análisis cualitativo de las manifestaciones y antecedentes clínicos registrados en las pacientes evaluadas en ambos grupos. En cuanto a la presencia de sintomatología clínica, las mujeres tanto de los casos como de los controles, en su mayoría se encontraban asintomáticas; mientras que las que si presentaban síntomas refirieron principalmente la presencia de leucorrea y muy pocas disuria o sangrado postcoital. Por su parte, al interrogar sobre algunos antecedentes clínicos predominó en las mujeres infértiles el antecedente de enfermedad pélvica inflamatoria (EPI), seguido de cervicitis e infección por el Virus del Papiloma Humano (VPH); en tanto que en las mujeres fértiles prevaleció en orden de frecuencia el antecedente de cervicitis, infección por VPH y EPI. Cabe destacar que sólo el antecedente de haber presentado un cuadro de EPI mostró significancia estadística $(p<0,05)$.

Respecto a las características de la esterilidad observada en el grupo de mujeres con alteraciones en su capacidad reproductiva presentadas en la Tabla III, se pudo evidenciar que predominó la infertilidad primaria en $63,33 \%$ de los casos, mientras que la infertilidad secundaria se observó solo en $36,67 \%$ de estas mujeres. En cuanto a la causas prevaleció el factor tubo-peritoneal en 53,33\% de las pacientes, seguidas de causas ovulatorias $(36,67 \%)$, uterinas $(6,67 \%)$ o debidas al factor cervical $(3,33 \%)$; cabe resaltar que en $9(30 \%)$ de los casos la causa de la infertilidad era mixta, es decir, debido a algún factor femenino en conjunto con el factor masculino.

Tabla I

CARACTERIZACIÓN DE LA MUESTRA DE MUJERES FÉRTILES E INFÉRTILES

\begin{tabular}{lccrr}
\hline Análisis/Alternativas & $\begin{array}{c}\text { GRUPO A } \\
\text { (Infértiles) } \\
\text { X } \pm \text { DE }\end{array}$ & $\begin{array}{c}\text { GRUPO B } \\
\text { (Fértiles) } \\
\text { X } \pm \text { DE }\end{array}$ & $\begin{array}{c}\mathrm{t} \\
\mathrm{p}^{*}\end{array}$ & $\begin{array}{c}\text { Valor } \\
\text { Edad }\end{array}$ \\
Menarquía & $37,23 \pm 6,45$ & $36,86 \pm 5,20$ & 0,244 & 0,807 \\
Sexarquía & $12,30 \pm 1,53$ & $12,43 \pm 1,22$ & 0,372 & 0,711 \\
Número de parejas & $19,87 \pm 4,07$ & $19,57 \pm 4,33$ & 0,189 & 0,851 \\
Años de convivencia con pareja actual & $02,33 \pm 1,40$ & $02,71 \pm 1,14$ & 0,801 & 0,429 \\
\hline
\end{tabular}

* Comparación de medias por T de Student con una significancia estadística $p<0,05$ 
Tabla II

MANIFESTACIONES Y ANTECEDENTES CLÍNICOS EN MUJERES FÉRTILES E INFÉRTILES

\begin{tabular}{lccccc}
\hline \multirow{2}{*}{ Análisis/Alternativas } & \multicolumn{2}{c}{$\begin{array}{c}\text { GRUPO A } \\
\text { (Infértiles) }\end{array}$} & \multicolumn{2}{c}{$\begin{array}{c}\text { GRUPO B } \\
\text { (Fértiles) }\end{array}$} & Valor p * \\
& $\mathrm{Fa}$ & $\mathrm{Fr}$ (\%) & $\mathrm{Fa}$ & $\mathrm{Fr}(\%)$ & \\
\hline Manifestaciones clínicas & & & & & \\
Asintomáticas & 18 & 60,00 & 21 & 70,00 & 0,588 \\
Disuria & 03 & 10,00 & 02 & 06,67 & 0,640 \\
Leucorreas & 07 & 23,33 & 06 & 20,00 & 0,754 \\
Sangrado postcoital & 02 & 06,67 & 01 & 03,33 & 0,553 \\
Antecedentes clínicos & & & & & \\
Cervicitis & & & & & \\
EPI & 09 & 30,00 & 12 & 40,00 & 0,588 \\
Ectopia cervical & 12 & 40,00 & 03 & 10,00 & 0,017 \\
ITS & 04 & 13,33 & 02 & 06,67 & 0,667 \\
VPH & 01 & 03,33 & 02 & 06,67 & 0,553 \\
Neoplasia intraepitelial & 07 & 23,33 & 09 & 30,00 & 0,770 \\
& 01 & 03,33 & 02 & 06,67 & 0,553 \\
\hline
\end{tabular}

* Chi cuadrado con una significancia estadística de $p<0,05$.

Tabla III

TIPOS Y CAUSAS DE INFERTILIDAD

\begin{tabular}{lcc}
\hline \multicolumn{1}{c}{ Análisis/Alternativas } & Fa & Fr (\%) \\
\hline Tipo de Infertilidad & & \\
Primaria & 19 & 63,33 \\
Secundaria & 11 & 36,67 \\
Causas de Infertilidad & & \\
Cervicales & 01 & 03,33 \\
Uterinas & 02 & 06,67 \\
Tubo-peritoneal & 16 & 53,33 \\
Ovulatorias & 11 & 36,67 \\
Mixtas (Factor masculino y femenino) & 09 & 30,00 \\
\hline
\end{tabular}

En la Tabla IV se presentan los resultados obtenidos del diagnóstico molecular del genoma de CT determinado mediante PCR, a partir de muestras de hisopados del canal endocervical de mujeres infértiles y fértiles; donde se detectó una prevalencia de infección por CT del 18\% $(n=11)$ en ambos grupos; sin embargo, hubo una mayor frecuencia del diagnóstico entre las mujeres infértiles $(26,67 \%$ vs. $10 \%$, respectivamente), aunque no significativa $(p>0,05)$. Por su parte, para UU se determinó una prevalencia de infección por este microorganismo del 35\%; siendo más frecuentemente detectado en los hisopados obtenidos de las mujeres infértiles $(46,67 \% ; n=14)$ que en los de las pacientes fértiles $(23,33 \% ; n=7)$, diferencia que resulto ser estadísticamente significativa $(p<0,05)$. Importa destacar

Tabla IV

DIAGNÓSTICO MOLECULAR DE CHLAMYDIA TRACHOMATIS Y UREAPLASMA UREALYTICUM EN MUJERES FÉRTILES E INFÉRTILES

\begin{tabular}{|c|c|c|c|c|c|c|}
\hline \multirow[t]{2}{*}{ Análisis/Alternativas } & \multicolumn{2}{|c|}{$\begin{array}{l}\text { GRUPO A } \\
\text { (Infértiles) }\end{array}$} & \multicolumn{2}{|c|}{$\begin{array}{l}\text { GRUPO B } \\
\text { (Fértiles) }\end{array}$} & \multirow[t]{2}{*}{$x^{2}$} & \multirow[t]{2}{*}{ Valor $\mathrm{p}^{*}$} \\
\hline & $\mathrm{Fa}$ & $\operatorname{Fr}(\%)$ & $\mathrm{Fa}$ & $\operatorname{Fr}(\%)$ & & \\
\hline \multicolumn{7}{|c|}{ Chlamydia trachomatis } \\
\hline Positivo & 08 & 26,67 & 03 & 10,00 & 1,781 & 0,182 \\
\hline Negativo & 22 & 73,33 & 27 & 90,00 & & \\
\hline \multicolumn{7}{|c|}{ Ureaplasma urealyticum } \\
\hline Positivo & 14 & 46,67 & 07 & 23,33 & 4,440 & 0,035 \\
\hline Negativo & 16 & 53,33 & 23 & 76,67 & & \\
\hline
\end{tabular}

* Chi cuadrado con una significancia estadística de $\mathrm{p}<0,05$. 
que en dos de los casos se encontró una co-infección entre CT y UU.

Los resultados obtenidos de la histerosalpingografía, realizada tanto a las mujeres infértiles como a las fértiles, se muestran en la Tabla $\mathrm{V}$. La evaluación de la permeabilidad de las trompas uterinas detectó una mayor frecuencia de permeabilidad tubárica en las pacientes fértiles que en las infértiles $(80 \%$ vs. $40 \%)$, siendo el compromiso tubárico mayor en las pacientes infértiles, donde se detectó mayormente la presencia de obstrucción bilateral de las trompas de Falopio $(p<0,05)$.

En la Tabla VI se presenta la correlación en- tre la valoración de la permeabilidad tubárica y la presencia de infección genital por CT o UU, análisis que evidencia que en las mujeres infértiles el diagnóstico de cualquiera de estos dos microorganismo aumenta casi 3 veces más la probabilidad de presentar obstrucción de las trompas uterinas, diferencia sin significancia estadística $(p>0,05)$. Por su parte, en las mujeres fértiles la presencia de UU en los hisopados endocervicales se asoció con un riesgo de 5 veces más probabilidad de obstrucción tubárica, el doble del riesgo de las mujeres infértiles positivos para esta bacteria, diferencias sin significancia estadística $(p>0,05)$.

Tabla V

RESULTADO DE LA HISTEROSALPINGOGRAFÍA EN MUJERES FÉRTILES E INFÉRTILES

\begin{tabular}{lcccccc}
\hline Análisis/Alternativas & \multicolumn{2}{c}{$\begin{array}{c}\text { GRUPO A } \\
\text { (Infértiles) }\end{array}$} & \multicolumn{2}{c}{ GRUPO B } & $x^{2}$ & $p^{*}$ \\
& Fa & Fr (\%) & Fa & Fr (\%) & & \\
\hline Permeable & 12 & 40,00 & 24 & 80,00 & 8,403 & 0,003 \\
Obstrucción unilateral & 07 & 23,33 & 06 & 20,00 & & \\
Obstrucción bilateral & 11 & 36,67 & - & - & & \\
\hline
\end{tabular}

* Chi cuadrado con una significancia estadística de $p<0,05$.

Tabla VI

ESTIMACIÓN DEL RIESGO DE OBSTRUCCIÓN TUBÁRICA EN MUJERES FÉRTILES E INFÉRTILES CON INFECCIÓN GENITAL

\begin{tabular}{lcccc}
\hline $\begin{array}{l}\text { Análisis/Alternativas } \\
\text { OBSTRUCCIÓN }\end{array}$ & $\begin{array}{c}\text { HSG } \\
\text { PERMEABILIDAD }\end{array}$ & $\begin{array}{c}\text { Odds Ratio * } \\
\text { (IC95\%) }\end{array}$ & Valor $\mathrm{p}^{* *}$ \\
\hline $\begin{array}{l}\text { Chlamydia trachomatis }(+) \\
\text { Infértiles }\end{array}$ & 06 & 02 & $\begin{array}{c}2,5 \\
(0,410-15,230)\end{array}$ & 0,555 \\
Fértiles & 01 & 02 & 0,294 & 0,542 \\
& & & $(0,023-3,671)$ & \\
$\begin{array}{l}\text { Ureaplasma urealyticum (+) } \\
\text { Infértiles }\end{array}$ & 10 & 04 & 2,5 & 0,411 \\
Fértiles & 03 & 04 & $(0,547-11,410)$ & 5 \\
& 0 & & $(0,727-34,346)$ & 0,235 \\
\hline
\end{tabular}

\footnotetext{
* Análisis de Regresión logística

${ }^{\star *}$ Chi cuadrado con una significancia estadística de $p<0,05$.
} 


\section{DISCUSIÓN}

Aunque el protocolo para la evaluación de la infertilidad en diferentes instituciones puede variar entre una clínica y otra, la mayoría de las clínicas de infertilidad incluyen como parte de la evaluación inicial de las enfermedades de transmisión sexual, pruebas para la detección de infecciones por CT, $\mathrm{UU}, \mathrm{MH}$ o NG, las cuales pudiesen estar interfiriendo con la alteración de la capacidad reproductiva; mediante la toma de muestras del tracto genital para cultivos y pruebas moleculares, o séricas para la detección de anticuerpos contra éstos gérmenes.

La alteración del factor tubo-peritoneal constituye una de las causa más frecuente de infertilidad $(6,24)$, tal como se evidenció en las mujeres infértiles valoradas, se conoce que la enfermedad inflamatoria pélvica es una de las condiciones patológicas más importantes que causan daño permanente y las adherencias que afectan a las trompas de Falopio $(25,26,27)$, antecedente significativo en estas pacientes $(p<0,05)$. Al respecto, patógenos como CT y NG han sido considerados los principales patógenos en provocar daño irreversible en la mucosa tubárica, aunque recientemente se han involucrado también en el daño tubárico a microorganismos de la familia Mycoplasmataceae como UU y MH (28).

En las trompas de Falopio, CT es responsable tanto de daños en los cilios como de la obstrucción de las mismas, ya que la repuesta inmune a la Chamydia produce cicatrización y fibrosis en el interior de las trompas uterina lo que aumenta el riesgo de embarazos ectópicos y explicaría la infertilidad (29), reportándose que está asociada a $2 / 3$ de los casos de infertilidad tubárica y a $1 / 3$ de los embarazos ectópicos (30). No obstante, los factores asociados con la evasión de Chlamydia por los mecanismos de defensa inmune y la migración desde la parte inferior a la superior del tracto genital femenino, siguen siendo incompletamente determinados (10).

Al igual que lo manifestado por otros autores, quienes han señalado que la infección por CT puede ser asintomática hasta en $80 \%$ de los casos (31), se observó una gran porcentaje mujeres que no referían presentar síntomas, observación que es sumamente preocupante ya que al haber una alta tasa de infecciones asintomáticas, estas mujeres por no presentar síntomas, no acuden a consultas médicas y por lo tanto, no son investigadas ni sometidas a tratamiento antimicrobiano (18). También hay pruebas suficientes de que la mayoría de las mujeres que sufren de infertilidad tubárica no tienen antecedentes de enfermedad pélvica inflamatoria sintomática (28) por lo que estando éstas infecciones del tracto genital relacionadas con la infertilidad tubárica, la presencia de infección subclínica podría pasar desapercibida y originar daño en la mucosa tubárica.

En este estudio se evidenció una prevalencia global de infección de CT del $18 \%$, aunque siendo mayor en las mujeres infértiles que en las fértiles $(26,67 \%$ vs. $10 \%)$, esta diferencia no mostró presentar significancia estadística, sin embargo, Urbina y cols (30), reportaron en mujeres con infertilidad una prevalencia general de CT diagnosticada por la determinación de anticuerpos séricos mediante ELISA de $25,40 \pm 6,26 \%$, similar a la encontrada en esta investigación. Por su parte, Siemer (16), determinó en mujeres infértiles una baja prevalencia de CT por PCR $(2,4 \%)$, pero una seroprevalencia significativamente más altos de anticuerpos específicos IgG (39\%) e IgA (14\%), lo cual sugiere que las infecciones anteriores por CT puede contribuir a la infertilidad; aunque es importante destacar que la detección de anticuerpos IgG anti-chlamydia como marcador diagnóstico de la infertilidad a pesar de haber demostrado una alta sensibilidad (72,7\%), tiene una baja especificidad $(44,4 \%)$.

No obstante, Wilkowska (32), consideran la detección de anticuerpos específicos anti-chlamydia un procedimiento de diagnóstico no invasivo valioso al evaluar la prevalencia de la infección urogenital por CT en mujeres con diagnostico de infertilidad, pues determinaron que la infección por CT es un factor etiológico muy importante de infertilidad femenina, ya que al comparar mujeres con infertilidad tubárica o infertilidad de otro origen determinaron en ambos grupos una prevalencia similar de CT detectada por PCR ( $8,7 \%$ vs. $8,3 \%)$, en tanto, que los anticuerpos específicos anti-Chlamydia tipo IgA e IgG prevalecieron significativamente en las mujeres con infertilidad tubárica $(13,0 \%$ vs. $6,3 \%$ y $39,1 \%$ vs. $10,4 \%$, IgA e IgG, respectivamente).

De igual forma, otros investigadores (9) han determinado que las pacientes con factor tubárico desarrollaron niveles significativamente más altos de anticuerpos contra CT; resultado que podría proporcionar información útil tanto para el desarrollo de una prueba de detección no invasiva para el factor tubárico como para la construcción de vacuna anti-CT. A su vez, en otra investigación realizada en Brasil (33) se encontró una alta prevalencia de la infección por CT en mujeres infértiles de $52,8 \%(n=56)$, con una asociación significativa del $5 \%(p<0,05)$ de la infección por clamidia con el ingreso familiar, bajo nivel socio-económico y desempleo $(p<0,05)$; al respecto, estudios epidemiológicos han señalado como factores de riesgo: ser una persona soltera sexualmente activa, bajo nivel socio-económico, tener múltiples compañeros sexuales, inicio temprano de las 
relaciones sexuales, usar anticonceptivos orales o dispositivos intrauterinos, la no utilización del preservativo, así como tener historia previa de ITS, flujo vaginal o dolor abdominal vago (30).

En este mismo orden de ideas, Omo (34) reportó una prevalencia de anticuerpos séricos contra CT significativamente mayor en mujeres infértiles $(65,8 \% ; p<0,001)$; sin embargo, no encontró una asociación fuerte e independiente entre los anticuerpos de CT y el riesgo de ser infértil. Aunque, otras investigaciones han concluido que las infecciones por CT prevalecen en parejas con infertilidad, pues se había encontrado un $96,8 \%$ de positividad en las parejas infértiles estudiadas (35), mientras que otras no han conseguido diferencias significativas entre las parejas fértiles e infértiles en términos de la prevalencia de las infecciones por CT o UU (36).

A su vez, al compararse mujeres con infertilidad tubárica y controles fértiles se ha detectado una prevalencia significativa $(p<0,05)$ de CT de $14,99 \%$, UU de $23,24 \%$ ) y $9,17 \%$, para la coinfección por $\mathrm{CT}$, UU y $\mathrm{MH}$; puesto que las tasas de infección en pacientes con infertilidad tubárica fueron superiores a las de mujeres fértiles, se ha reportado que la infección por estos microorganismos tiene cierta relevancia en la infertilidad tubárica (37). CT también ha resultado ser el microorganismo más frecuente en mujeres infértiles y abortadoras habituales $(45,2 \%)$, seguido por UU y la asociación entre CT-UU (7); sin embargo, Casari y cols (8), no encontraron diferencias significativas entre las mujeres fértiles sintómaticas y las asintomáticas con problemas de fertilidad en cuanto a la presencia de CT $(2,15 \%$ vs. $0,51 \%$, respectivamente) o UU $(4,86 \%$ vs. $3,79 \%$, respectivamente).

Respecto a UU, aunque algunos autores $(38,39)$ lo consideran un comensal del tracto genital, Imudia y cols (23) encontraron una prevalencia del $20,1 \%$ de pacientes positivos para UU durante la evaluación inicial de mujeres infértiles, aunque existen algunas dudas de su papel en la infertilidad (40). En la presente investigación se encontró significativamente una mayor prevalencia de este germen en las mujeres infértiles $(p<0,05)$, contrario a los hallazgos reportados en otro estudio, donde se encontró que la infección más común en el canal cervical al comparar pacientes infértiles y controles fértiles fue UU, pero sin diferencias significativas $(p=0,602)$ entre los grupos (41).

Así mismo, los resultados obtenidos por diversos estudios señalan que el papel de los micoplasmas genitales en la infertilidad humana todavía no está claro y requieren más investigaciones, puesto que en otros hay resultados controvertidos que no confirman un papel patogénico definitivo para este agente; en tal sentido, otros investigadores $(22,42)$ no han encontrado diferencias significativas entre mujeres fértiles e infértiles para la presencia de UU. En tanto que Costoya y cols (28) al analizar muestras de lavados tubáricos obtenidos por laparoscopia en mujeres infértiles y controles sanos, encontraron que UU fue el microorganismo más frecuente aislado en ambos grupos, con una diferencia significativa entre los casos y controles, con una mayor incidencia entre los segundos, lo que indica que UU es capaz de crecer en las trompas de Falopio sanas como un microorganismo comensal. Precisamente, la dificultad de aceptar a $\mathrm{MH}$ y UU, como causa de enfermedad, se debe tanto a que los organismos se recuperan de sujetos asintomáticos como a que las muestras no pueden ser obtenidas fácilmente del sitio infectado (43).

Es por ello que algunas investigaciones no recomiendan, durante la evaluación inicial de las parejas infértiles, la detección rutinaria de los mycoplasmas (UU y $\mathrm{MH}$ ) sin ninguna evidencia clínica, dado a que no es rentable debido a bajas tasas de detección $(23,36,44)$. No obstante, la infección por este microorganismo puede reducir la tasa de éxito de los tratamientos de infertilidad altamente especializados, entre ellos la inseminación artificial intrauterina, la transferencia de gametos, la fertilización in vitro o la inyección intracitoplasmática de espermatozoides; asimismo, podría causar una marcada reducción del desarrollo embrionario temprano posterior a la fertilización in vitro (22).

Sin embargo, con relación a la CT, la demostración de la colonización cervical de CT por técnicas moleculares puede ser un método prometedor para la detección de la infección pélvica asintomática en pacientes con infertilidad inexplicada (23); asimismo, se ha señalado la necesidad de implementar un monitoreo de rutina para su diagnóstico, incluyendo su determinación en el control ginecológico anual, a fin de prevenir que la infección pase a ser crónica (30).

Por consenso se recomienda realizar una pesquisa anual de CT en las adolescentes y mujeres jóvenes menores de 25 años sexualmente activas, mientras que debe realizarse en todas las embarazadas independientemente de la edad y en las mujeres mayores de 26 años, sólo si presentan factores de riesgo como un nuevo o múltiples compañeros sexuales $(45,46)$; las cuales representan estrategias importantes para disminuir la prevalencia de esta infección y de sus secuelas, como el embarazo ectópico y la infertilidad tubárica. Al parecer las personas más vulnerables son las mujeres jóvenes con edades comprendidas entre 15 y 24 
años, probablemente por la característica anatómica de tener la unión escamoso-columnar del cérvix expuesta, lo que favorece la infección (30).

Para ello, técnicas de amplificación de ácidos nucleícos por medio de la reacción en cadena de la polimerasa muestran una excelente sensibilidad y especificidad (47), permitiendo detectar hasta $30 \%$ más de muestras positivas que con otros procedimientos y no requieren de una toma de muestra invasora, lo cual las convierte en las técnicas de referencia para diagnóstico, especialmente en mujeres, que suelen ser asintomáticas (48). Si bien el diagnóstico de laboratorio tradicional para las infecciones causadas por CT es el aislamiento en cultivos celulares, este requiere condiciones estrictas de recolección y transporte, como también de personas expertas y equipos costosos (49), asimismo, la detección de anticuerpos, a pesar de ser un ensayo efectivo, no invasivo y viable para ser realizado en países en desarrollo, muestra una menor sensibilidad (50).

Si bien el tipo y diseño de la investigación: comparativo, correlacional y transeccional, presenta algunas limitaciones para efectuar un análisis de causalidad directa y no excluye otros efectos que pudiesen explicar la fertilidad, los resultados del trabajo informa de una considerable prevalencia de estos patógenos en la muestra evaluada. Asimismo, el estudio tiene limitaciones para que los resultados sean generalizables, puesto que se realizó un muestreo intencionado, por tanto, sería recomendable continuar el mismo, incluyendo una mayor cantidad de mujeres seleccionadas mediantes criterios más rigurosos, además de investigar tanto los anticuerpos contra estos microorganismos de modo de poder conocer la prevalencia de infecciones crónicas, como de aplicar estas técnicas de diagnóstico molecular en sus parejas. Se pudo evidenciar que en las mujeres infértiles el diagnóstico tanto de CT como UU aumenta casi 3 o 5 veces más la probabilidad de presentar obstrucción de las trompas uterinas, respectivamente, aunque este riesgo más elevado no mostró significancia estadística $(p>0,05)$.

\section{CONCLUSIÓN}

Gran parte de las mujeres con problemas de fertilidad presentan infección por CT o UU, patógenos de transmisión sexual que pudiesen tener responsabilidad en el daño tubárico y explicar su infertilidad.

\section{REFERENCIAS}

1. Parada L. Infertilidad y pareja: construcciones narrativas como horizonte para la intervención. Revista Diversitas - Perspectivas en Psicología 2006;2:149-58.

2. Brugo $S$, Chillik $C$, Kopelman S. Definición y causas de la infertilidad. Rev Col Obstet Ginecol 2003;54:227-48.

3. Burns LH. Psychiatric aspects of infertility and infertility treatments. Psychiatr Clin N Am 2007;30:689-716.

4. Williams C, Giannopoulos T, Sherriff EA. Investigation of infertility with the emphasis on laboratory testing and with reference to radiological imaging. J Clin Pathol 2003;56:261-7.

5. Jose-Miller AB, Boyden JW, Frey KA. Infertility. Am Fam Physician 2007;75:849-56.

6. Wiesenfeld HC, Hillier SL, Meyn LA, Amortegui AJ, Sweet RL. Subclinical pelvic inflammatory disease and infertility. Obstet Gynecol 2012;120:37-43.

7. Ortiz CE, Hechavarría CE, Ley M, Álvarez G, Hernández Y. Estudio de Chlamydia trachomatis, Ureaplasma urealyticum y Mycoplasma hominis en pacientes infértiles y abortadoras habituales. Rev Cubana Obstet Ginecol 2010;36:73-84.

8. Casari E, Ferrario A, Morenghi E, Montanelli A. Gardnerella, Trichomonas vaginalis, Candida, Chlamydia trachomatis, Mycoplasma hominis and Ureaplasma urealyticum in the genital discharge of symptomatic fertile and asymptomatic infertile women. New Microbiologica 2010;33:69-76.

9. Rodgers AK, Wang J, Zhang Y, Holden A, Berryhill $B$, Budrys NM, et al. Association of tubal factor infertility with elevated antibodies to Chlamydia trachomatis caseinolytic protease P. Am J Obstet Gynecol 2010;203:494e7-494e14.

10. Soto CS. Infección por Chlamydia trachomatis asociado a factor tubárico de subfertilidad: aspectos inmunogenéticos. Waxapa 2010;3:43-59.

11. Donnellan NM, Wiesenfeld HC. Inflammation on liquid-based cervical cytology: can leukocytes be used to triage for Chlamydia trachomatis testing? Am J Obstet Gynecol 2007;196:e33-e35.

12. Centers for Disease Control and Prevention. Trends in Reportable Sexually Transmitted Disease Surveillance in the United States 2005. National Surveillance Data for Chlamydia, and Syphilis. 2006. [Documento en línea]. Disponible: http//www.cdc.gov/STD/stats. Fecha de consulta: 21 de Mayo de 2012.

13. American College of Obstetricians and Gynecologists. Primary and preventive care: Periodic assessments. Obstet Gynecol 2006;357:1615-22.

14. Sánchez RM, Ruiz Al, Ostos OL. Prevalencia de Chlamydia trachomatis detectada por reacción en cadena de la polimerasa en un grupo de mujeres jóvenes sintomáticas y asintomáticas en Bogotá, Colombia. Rev Col Obstet Ginecol 2006;57:171-81.

15. Arráiz N, Marcucci R, Colina S, Reyes F, Rondón N, Bermúdez $\mathrm{V}$, et al. Infección por Chlamydia trachomatis en mujeres consultantes en Maracaibo, Venezuela. Rev Salud Pública 2008;10:615-24. 
16. Siemer J. Chlamydia trachomatis infection as a risk factor for infertility among women in Ghana, West Africa. Am J Trop Med Hyg 2008;78:323-7.

17. Frontela $\mathrm{M}$, Rodríguez $\mathrm{Y}$, Verdejas OL, Valdés FJ. Infección por Chlamydia trachomatis en mujeres cubanas en edad reproductiva. Rev Cubana Endocrinol 2006; 17 [Periódico en línea]. Disponible:http://www.imbiomed.com.mx/1/1/articulos. php? method=showDetail\&id_articulo $=38458 \&$ id_ seccion=705\&id_ejemplar=3967\&id_revista=58. Fecha de consulta: 04 de Junio de 2012.

18. Arráiz N, Ginestre M, Perozo A, Castellano M, Urdaneta B, García MM. Diagnóstico molecular y prevalencia de infecciones por Chlamydia trachomatis en pacientes sintomáticas y asintomáticas de una población del estado de Zulia, Venezuela. Rev Chil Infect 2007;24:48-52.

19. Arráiz N, Ginestre M, Perozo A, Castellano M, Urdaneta V. Detección de Chlamydia trachomatis en muestras de hisopado endocervical por inmunofluorescencia directa y reacción en cadena de la polimerasa. Revi Soc Venezolana Microbiol 2006;26:14-8.

20. Arráiz N, Marcucci R, Urdaneta B, Colina S, Romero $Z$. Diagnóstico molecular en la evaluación de infecciones urogenitales por Chlamydia trachomatis. Rev Obstet Ginecol Venez 2008;68:195-201.

21. Castellano M, Ginestre M, Perozo A, Alaña F, Fernández M, Rincón G. Colonización vaginal por micoplasmas genitales en mujeres embarazadas y no embarazadas. Invest Clin 2007;48:419-29.

22. Fenkci V, Yilmazer M, Aktepe OC. Have Ureaplasma urealyticum and Mycoplasma hominis infections any significant effect on female fertility? Infez Med 2002;10:220-3.

23. Imudia AN, Detti L, Puscheck EE, Yelian FD, Diamond MP. The prevalence of ureaplasma urealyticum, mycoplasma hominis, chlamydia trachomatis and neisseria gonorrhoeae infections, and the rubella status of patients undergoing an initial infertility evaluation. J Assist Reprod Genet 2008;25:43-6.

24. Brassard M, AinMelk Y, Baillargeon JP. Basic infertility including polycystic ovary syndrome. Med Clin N Am 2008;92:1163-92.

25. Huddleston HG Cedars MI Sohn SH Giudice LC Fujimoto VY. Racial and ethnic disparities in reproductive endocrinology and infertility. Am J Obstet Gynecol 2010;202:413-9.

26. Per-Anders M. Tubal factor infertility, with special regard to chlamydial salpingitis. Curr Opin Infect Dis 2004; 17:49-52.

27. Guerra F, Flores S, Arteaga G, Zamora A, López $M$, Ortiz FJ. Factores de riesgo y secuelas reproductivas asociados a la infección por Chlamydia trachomatis en mujeres infértiles. Salud Pública Méx 2003;45(Supl.5):S672-S680.

28. Costoya A, Morales F, Borda P, Vargas R, Fuhrer J, Salgado N, et al. Mycoplasmateceae species are not found in Fallopian tubes of women with tubo-peritoneal infertility. Braz J Infect Dis 2012;16:273-8.

29. Baraitser P, Alexander S, Sheringham J. Chlamydia trachomatis screening in young women. Curr Opin Obstet Gynecol 2011;23:315-20.
30. Urbina MT, Medina R, Muñoz G, Sánchez V, Benjamín I, Lerner J. Infección por Chlamydia trachomatis. Rev Obstet Ginecol Venez 2010;70:90-6.

31. Cervantes E. Infecciones causadas por Chlamydia trachomatis. Rev Fac Med UNAM 2009;52:18-22.

32. Wilkowska M. Chlamydia trachomatis urogenital infection in women with infertility. Adv Med Sci 2009;54:825.

33. De Lima NS. High prevalence detection of Chlamydia trachomatis by polymerase chain reaction in endocervical samples of infertile women attending university hospital in Manaus-Amazonas, Brazil. Gynecol Obstet Invest 2011;72:220-6.

34. Omo LO. Association of Chlamydia trachomatis serology with tubal infertility in Nigerian women. J Obstet Gynaecol Res 2007;33:688-95.

35. Preciado R, Arredondo RR, García A, Manterola D, Blanco N, Martínez JC. Identificación de Chlamydia trachomatis en parejas infértiles. Rev Mex Reprod 2011;4:72-6.

36. Günyeli I, Abike F, Dünder I, Aslan C, Tapısız OL, Temizkan O, Payaslı A, Erdemoğlu E. Chlamydia, Mycoplasma and Ureaplasma infections in infertile couples and effects of these infections on fertility. Arch Gynecol Obstet 2011;283:379-85.

37. Zhou Y, Xu XL, Wang CP, Zhou M, Zeng XH. Detection and the antibiotic susceptibility analysis of mycoplasma and chlamydia in urogenital tract infections of 327 cases patients with tubal infertility. Zhonghua Shi Yan $\mathrm{He}$ Lin Chuang Bing Du Xue Za Zhi 2011;25:201-4.

38. Mayo DM, Barrios E, Ruiz R, Cedillo L, Rivera JA. Aislamiento de mollicutes en faringe y tracto urogenital. Enf Inf Microbiol 2009;29:6-10.

39. Núñez JT. Mycoplasma hominis y Ureaplasma urealyticum en diferentes patologías ginecológicas. Invest Clin 1999;40:9-24.

40. Roca B. Infecciones por micoplasmas. Rev Clin Esp 2006;206:239-42.

41. Guven MA, Dilek U, Pata O, Dilek S, Ciragil P. Prevalance of Chlamydia trochomatis, Ureaplasma urealyticum and Mycoplasma hominis infections in the unexplained infertile women. Arch Gynecol Obstet 2007;276:219-23.

42. Grześko J, Elias M, Maczyńska B, Kasprzykowska $U$, Tłaczała M, Goluda M. Frequency of detection of Ureaplasma urealyticum and Mycoplasma hominis in cervical canal and the Douglas pouch of infertile and fertile women. Med Dosw Mikrobiol 2007;59:169-75.

43. Cervantes E. Micoplasmas patógenos para el humano. Rev Fac Med UNAM 2009;52:253-9.

44. Rosemond A, Lanotte P, Watt S, Sauget AS, Guerif F, Royère $D$, et al. Systematic screening tests for Chlamydia trachomatis, Mycoplasma hominis and Ureaplasma urealyticum in urogenital specimens of infertile couples. Pathol Biol (Paris) 2006;54:125-9.

45. Tao G, Hoover KW, Kent CK. 2009 Cervical cytology guidelines and Chlamydia testing among sexually active young women. Obstet Gynecol 2010;116:1319-23.

46. Hoover KW, Tao G. Missed opportunities for Chlamydia screening of young women in the United States. Obstet Gynecol 2008;111:1097-102.

47. Sánchez V, Torres AE, Villalba JD. Diagnóstico de 
infección por Chlamydia trachomatis mediante PCR en pacientes que acuden a la Clínica de Especialidades de la Mujer de la Secretaría de la Defensa Nacional. Ginecol Obstet Mex 2009;77:13-8.

48. Martínez MA. Diagnóstico microbiológico de las infecciones de transmisión sexual (ITS). Parte 1. ITS no virales. Rev Chil Infect 2009;26:529-39.
49. Alfieri A, Ramírez LG, Arcila N, Guevara Y. Determinación de anticuerpos contra Chlamydia trachomatis en pacientes del Servicio de Infertilidad del Centro Médico "Dr. Rafael Guerra Méndez", Valencia, Venezuela. Rev Soc Ven Microbiol 2005;25:47-9.

50. Malik A. Chlamydia trachomatis infection in women with secondary infertility. Fertil Steri 2009;91:91-5. 